\title{
PREPARAÇÃO DE COMPOSTOS LAMELARES: SÍNTESE DO HIDROGENOFOSFATO DE ZIRCÔNIO CRISTALINO E SUA INTERCALAÇÃO COM AMINAS. UM EXPERIMENTO PARA ESTUDANTES DE GRADUAÇÃO
}

\author{
William da Silva Cardoso e Yoshitaka Gushikem* \\ Instituto de Química, Universidade Estadual de Campinas, CP 6154, 13084-971 Campinas - SP
}

Recebido em 23/4/04; aceito em 15/10/04; publicado na web em 17/2/05

\begin{abstract}
PREPARATION OF LAMELLAR COMPOUNDS: SYNTHESIS OF THE CRYSTALLINE ZIRCONIUM HYDROGENPHOSPHATE AND ITS INTERCALATION WITH AMINES. AN EXPERIMENT FOR UNDERGRADUATE STUDENTS. The present paper describes the synthesis of crystalline zirconium hydrogen phosphate by direct precipitation and its intercalation with pyridine and n-butylamine. The simple experiment was tested in the undergraduate inorganic chemistry laboratory course for chemistry students at IQ-UNICAMP using inexpensive reagents. The materials were characterized by powder X-ray diffraction and infrared analyses in order to obtain detailed information of the solid structure changes as a result of the intercalation process. Pyridine and n-butylamine are focused in this work as clear and elucidative examples leading to acid-base interactive processes that result in the well-formed infinite sequence of inorganic lamellar structures.
\end{abstract}

Keywords: intercalation; $\alpha$-zirconium hydrogenphosphate; undergraduate experiment.

\section{INTRODUÇÃO}

No estado sólido existem muitos compostos chamados de compostos lamelares ou bidimensionais, que se caracterizam pelo fato dos átomos que os constituem, ligados por forças de natureza covalente, estarem arranjados de modo a formar camadas ou lamelas $^{1-5}$. Em alguns compostos estas camadas são eletricamente neutras e estão unidas entre si por forças fracas do tipo van der Waals. Assim sendo, as forças que mantém as lamelas unidas (forças interlamelares) são bem mais fracas que as forças existentes entre os átomos presentes na lamela (forças intralamelares), causando uma forte anisotropia ao composto ${ }^{6}$. Tal situação possibilita que espécies como íons, átomos ou moléculas entrem no espaço interlamelar, sendo dado a este fenômeno o nome de intercalação ${ }^{7}$.

As intercalações são geralmente acompanhadas por um aumento na distância interlamelar da matriz hospedeira (composto lamelar), para que haja uma perfeita acomodação da espécie convidada (espécie que se liga no espaço interlamelar) ${ }^{8-10}$. O interesse nas reações de intercalação está exatamente no fato de que elas modificam as propriedades das espécies do novo material formado e tais modificações surgem da alteração na densidade eletrônica entre as espécies envolvidas. Restrições geométricas e espaciais são impostas a ambas. A presença de íons, átomos ou moléculas entre os planos basais da espécie hospedeira certamente terá um impacto sobre as propriedades físicas em seu "bulk", incluindo densidade, condutividade e características ópticas ${ }^{6}$.

Um considerável número de compostos inorgânicos de características cristalinas, tais como grafita, argilas, fosfatos, fosfonatos, arsenatos, oxicloretos, sulfetos metálicos, óxidos de metais de transição, etc., desperta elevado interesse em virtude das propriedades químicas inerentes, destacando-se dentre elas, os comportamentos relacionados às trocas iônicas de grupos ácidos da superfície e o processo de intercalação que ocorre no interior da lamela ${ }^{11-15}$.

Um interesse maior tem sido dado aos estudos realizados com

*e-mail: gushikem@iqm.unicamp.br o hidrogenofosfato de zircônio, $\mathrm{Zr}\left(\mathrm{HPO}_{4}\right)_{2} \cdot \mathrm{H}_{2} \mathrm{O}$, aqui simplificado pela representação $\alpha$-ZrP, por possuir um número maior de propriedades quando comparado com outros compostos de intercalação. Os compostos de intercalação do $\alpha$-ZrP são caracterizados por sua notável estabilidade. As moléculas hóspedes ou convidadas ficam retidas tão firmemente entre as camadas que sua remoção é difícil até no vácuo, em temperaturas superiores a $100{ }^{\circ} \mathrm{C}$. Além de sua habilidade para intercalar moléculas neutras entre suas camadas, tem-se conhecimento que seus prótons algumas vezes são susceptíveis à reação de troca catiônica numa faixa de $\mathrm{pH}$ variando entre 4 e 8, particularmente com íons de metais alcalinos e cátions orgânicos de cadeias longas ${ }^{16-18}$.

A investigação da química de intercalação deve ser feita de modo que o sistema em estudo seja bem caracterizado em todos os seus aspectos: composição, estrutura e natureza da ligação hospedeiro-convidado. Dentre as técnicas existentes, a técnica de difração de raios-X é importante na investigação dos aspectos estruturais dos compostos de intercalação. Através dela, pode-se acompanhar uma reação de intercalação, monitorando o aumento da distância interlamelar, pelo deslocamento dos picos referentes aos planos orientados na direção do eixo de empilhamento. Outra técnica que pode ser útil para esta finalidade é a espectroscopia vibracional, porque alguns modos normais de vibração da molécula convidada podem ser modificados após a intercalação.

No presente trabalho pretende-se apresentar um experimento simples, conduzido num laboratório de ensino de química para graduação, abordando a síntese de um composto inorgânico, especificamente a preparação do fosfato de zircônio cristalino e, em seguida, alterar suas propriedades pela intercalação com aminas neutras: piridina e n-butilamina.

As mudanças de propriedades do composto intercalado foram acompanhadas pelas técnicas de difração de raios-X e espectroscopia vibracional na região do infravermelho.

A estratégia do experimento compreende duas aulas práticas (duração de $8 \mathrm{~h}$, cada aula): na primeira, será feita a síntese do composto lamelar e na segunda aula, a intercalação com aminas e o estudo de suas propriedades. Os resultados apresentados foram 
obtidos pelos alunos do curso de Química Inorgânica Experimental II, oferecido pelo Instituto de Química da Unicamp, no primeiro semestre de 2003.

\section{PARTE EXPERIMENTAL}

\section{Síntese do fosfato de zircônio cristalino $(\alpha-\mathrm{ZrP})$}

$\mathrm{O} \alpha-\mathrm{ZrP}$ foi preparado de acordo com o método descrito na literatura $^{19}$. Dentro de uma capela, dissolveu-se $5,5 \mathrm{~g}$ de $\mathrm{ZrOCl}_{2} .8 \mathrm{H}_{2} \mathrm{O}(17,1 \mathrm{mmol}$ ) (Merck) em $80 \mathrm{~mL}$ de água destilada, em um béquer de plástico. À solução adicionou-se $4 \mathrm{~mL}$ de $\mathrm{HF}$ $40 \%$ (Nuclear) (Cuidado ao manusear HF) e $46 \mathrm{~mL}$ de $\mathrm{H}_{3} \mathrm{PO}_{4} 85 \%$ (Vetec). Aqueceu-se a mistura em banho-maria por $3 \mathrm{~h}$, à temperatura controlada de $80{ }^{\circ} \mathrm{C}$, agitando eventualmente até a evaporação do HF, observado pelo turvamento da solução e início da formação de cristais de cor branca (hidrogenofosfato de zircônio). Após $3 \mathrm{~h}$ de reação, observou-se, ao fundo do béquer, a deposição dos cristais do hidrogenofosfato de zircônio. Então, o sistema foi resfriado, usando banho de gelo. A suspensão foi filtrada em funil de Büchner e o sólido obtido foi lavado com água destilada até neutralização do $\mathrm{pH}$, em seguida foi seco à temperatura ambiente. $\mathrm{O}$ rendimento médio da síntese é de 60 a $70 \%$ quando o experimento é feito tomando-se todos os cuidados, conforme indica o roteiro.

\section{Intercalação de piridina e n-butilamina no $\alpha$-ZrP}

A intercalação das aminas foi feita pela adição, separadamente, de $50 \mathrm{~mL}$ de uma solução $0,1 \mathrm{~mol} \mathrm{~L}^{-1}$ de piridina (Nuclear) e nbutilamina (Nuclear) a porções de $0,5 \mathrm{~g}$ de $\alpha$-ZrP. As suspensões, mantidas em béqueres vedados, foram deixadas em repouso, à temperatura ambiente, por 7 dias. Transcorrido este tempo, as suspensões foram filtradas em funil de Büchner, os sólidos retidos foram lavados com água destilada até neutralização do $\mathrm{pH}$, em seguida os sólidos foram secos à temperatura ambiente.

As análises de difração de raios-X foram feitas no difratômetro de raios-X Shimadzu XRD 6000, utilizando como fonte de radiação a linha de emissão do cobre $(\mathrm{Cu} \mathrm{K} \alpha, \lambda=0,154 \mathrm{~nm})$, com voltagem de aceleração do tubo de emissão de cobre de $40 \mathrm{kV}$, corrente de $30 \mathrm{~mA}$ e velocidade de varredura de $10^{\circ} 2 \theta \mathrm{min}^{-1}$.

Os espectros de absorção na região do infravermelho foram obtidos com uso de pastilhas de $\mathrm{KBr}$ (1\% em massa da amostra), usando o espectrofotômetro BOMEM Hartmann \& Braun MBseries, com uma resolução de $4 \mathrm{~cm}^{-1}$ e 50 varreduras.

\section{CUIDADOS}

O ácido fluorídrico é uma substância que se apresenta na forma líquida incolor, de odor irritante, corrosiva e que ataca o vidro. É altamente tóxico por inalação, ingestão e contato com a pele. O ácido fosfórico é um líquido incolor xaroposo, corrosivo e em contato com os olhos ou a pele pode causar irritação e queimaduras severas. Respirar os vapores do ácido fosfórico pode irritar o nariz, a garganta e os pulmões, causando tontura e falta de ar.

\section{RESULTADOS E DISCUSSÃO}

$\mathrm{O}$ hidrogenofosfato de zircônio cristalino $\left(\mathrm{Zr}\left(\mathrm{HPO}_{4}\right)_{2} \cdot \mathrm{H}_{2} \mathrm{O}\right)$ foi obtido pelo método da precipitação direta de sais de $\mathrm{Zr}(\mathrm{IV})\left(\left(\mathrm{PO}_{4} /\right.\right.$ $\mathrm{Zr})=2$ ). Inicialmente o $\mathrm{ZrOCl}_{2} \cdot 8 \mathrm{H}_{2} \mathrm{O}$ é complexado com ácido fluorídrico, conforme mostra a reação abaixo ${ }^{20}$ :

$\mathrm{Zr}^{4+}+6 \mathrm{~F}^{-} \underset{\Delta}{\longrightarrow}\left[\mathrm{ZrF}_{6}\right]^{2-}$
O ácido fluorídrico é um excelente agente complexante para zircônio. A reação do complexo formado com o ácido fosfórico resultará no fosfato de zircônio cristalino, de acordo com a reação 20 :

$\left[\mathrm{ZrF}_{6}\right]^{2-}+6 \mathrm{H}^{+}+2 \mathrm{HPO}_{4}^{2-}+\mathrm{H}_{2} \mathrm{O} \underset{\Delta}{\longrightarrow} \mathrm{Zr}\left(\mathrm{HPO}_{4}\right)_{2} \cdot \mathrm{H}_{2} \mathrm{O}+6 \mathrm{HF}$

Quando a concentração de HF é suficientemente alta, há uma inibição da precipitação provocada pelo ácido fosfórico. Entretanto, quando a concentração de íons fluoreto é diminuída (por exemplo, pela evaporação de HF) o complexo formado, hexafluorzirconato, gradualmente dissocia-se e o hidrogenofosfato de zircônio começa a precipitar. A formação do hidrogenofosfato de zircônio a partir do complexo hexafluorzirconato é controlada pela velocidade de evaporação do HF. A velocidade de formação, extremamente lenta, permite a precipitação direta do composto cristalino e o crescimento de cristais com tamanho relativamente grande $(\geq 1,2 \mathrm{~mm})^{20}$.

A estrutura cristalina do hidrogenofosfato de zircônio na forma $\alpha-Z r P$ é composta por um plano de átomos do metal ligados entre si por pontes de ânions fosfatos, formando uma cadeia inorgânica infinita, cujo conjunto de átomos define um plano, também denominado lamela. Cada átomo metálico nesta lamela está octaedricamente coordenado com seis grupos fosfato. Na estrutura formada dos quatro oxigênios de cada fosfato, três deles estão ligados a três diferentes átomos do metal. Logo, o oxigênio remanescente liga-se a um próton que se posiciona dentro do espaço lamelar, ficando então disponível para atuar diretamente com base para estabelecer ligações, no composto intercalado ${ }^{16}$ (Figura 1).

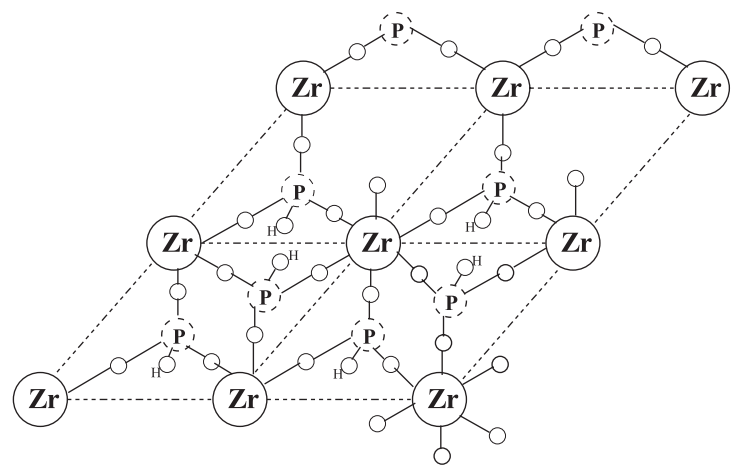

Figura 1. Esquema representativo da estrutura lamelar do hidrogenofosfato de zircônio cristalino. A camada idealizada na estrutura do $\alpha-\mathrm{Zr}\left(\mathrm{HPO}_{4}\right)_{2}$ relaciona a célula pseudohexagonal (linha tracejada) à célula monoclínica (linha sólida)

A Tabela 1 apresenta os resultados dos cálculos da distância interlamelar, para o hidrogenofosfato de zircônio cristalino e intercalado com piridina e n-butilamina, que foram feitos a partir dos dados obtidos dos difratogramas de raios-X (Figura 2) e pelo uso da lei de Bragg:

$2 \mathrm{~d} \operatorname{sen} \theta=\mathrm{n} \lambda$

onde, $\mathrm{d}=$ distância interlamelar; $\theta=$ ângulo de incidência; $\mathrm{n}=$ ordem de reflexão e $\lambda=$ comprimento de onda da radiação incidente.

$\mathrm{O}$ fosfato de zircônio $(\alpha-\mathrm{ZrP})$ obtido pelo método de precipitação é cristalino. As principais linhas de difração, identificadas na Figura 2a, são: $11,74^{\circ}$ (100\% de intensidade); $19,93^{\circ} ; 25,25^{\circ}$; e $34,15^{\circ}$. A partir dos dados referentes à linha de difração de maior intensidade, $2 \theta=11,74^{\circ}$, calculou-se o valor da distância interlamelar, d, que foi de 7,53 $\AA$ (Tabela 1), Este resultado é concor- 
Tabela 1. Picos de difração de raios-X com os respectivos valores de distância interlamelar calculados para $\alpha$-ZrP cristalino, $\alpha$-ZrP intercalado com piridina e $\alpha$-ZrP intercalado n-butilamina

\begin{tabular}{|c|c|c|c|c|c|}
\hline \multicolumn{2}{|c|}{$\alpha-\mathrm{ZrP}$} & \multicolumn{2}{|c|}{$\alpha-\mathrm{ZrP}+$ Piridina } & \multicolumn{2}{|c|}{$\alpha \mathrm{a}-\mathrm{ZrP}+\mathrm{n}$-butilamina } \\
\hline $2 \theta /$ grau & $\mathrm{d} / \AA$ & $2 \theta /$ grau & $\mathrm{D} / \AA$ & $2 \theta / \mathrm{grau}$ & $\mathrm{d} / \AA$ \\
\hline 11,74 & 7,53 & 8,00 & 11,04 & 4,83 & 18,27 \\
\hline 19,93 & 4,45 & 16,05 & 5,51 & 9,56 & 9,24 \\
\hline 25,25 & 3,52 & 23,53 & 3,78 & 14,18 & 6,24 \\
\hline 34,15 & 2,62 & 29,13 & 3,06 & - & - \\
\hline
\end{tabular}

dante com o da literatura ${ }^{16}$. Comparando o valor de $2 \theta=11,74^{\circ}$, com os existentes na literatura (Fichas no 19-1489, 21-1495 e 22$1022)^{21}$ para o $\mathrm{Zr}\left(\mathrm{HPO}_{4}\right)_{2} \cdot \mathrm{H}_{2} \mathrm{O}\left(2 \theta=11,72^{\circ}\right)$ e para o $\mathrm{Zr}\left(\mathrm{HPO}_{4}\right)_{2} \cdot 2 \mathrm{H}_{2} \mathrm{O}\left(2 \theta=7,20^{\circ}\right)$ pode-se propor que o $\alpha-\mathrm{ZrP}$ obtido no experimento se trata do hidrogenofosfato de zircônio monoidratado de sistema monoclínico.

A Figura $2 b$ mostra um difratograma de raios-X para o $\alpha \mathrm{ZrP}$ intercalado com piridina, sendo as principais linhas de difração as seguintes: $8,00^{\circ}$ (100\% de intensidade); $16,05^{\circ} ; 23,53^{\circ}$; e $29,13^{\circ}$. $\mathrm{Na}$ Figura $2 \mathrm{c}$ observa-se as principais linhas de difração do $\alpha-\mathrm{ZrP}$ intercalado com n-butilamina que são $4,83^{\circ}$ (100\% de intensidade); $9,56^{\circ}$; e $14,18^{\circ}$.
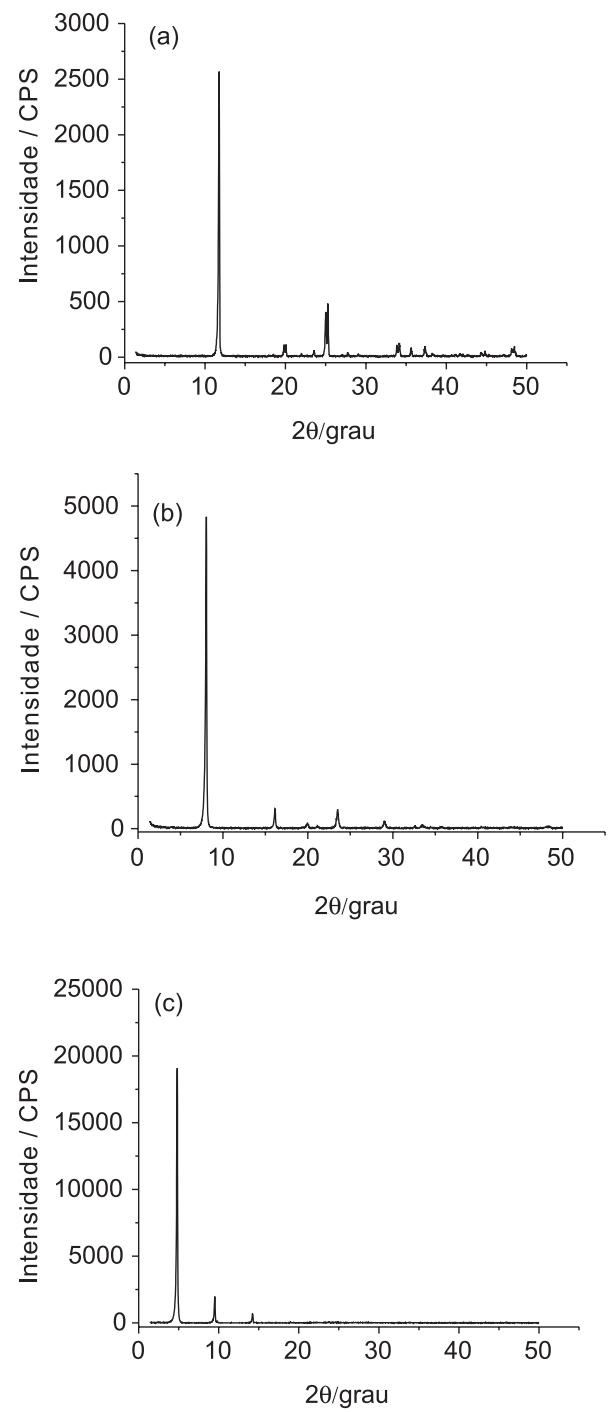

Figura 2. Difratogramas de raios- $X$ do hidrogenofosfato de zircônio (a) cristalino, (b) intercalado com piridina e (c) intercalado com n-butilamina
A partir dos dados referentes às linhas de difração de maior intensidade, $2 \theta=8,00^{\circ}$ para a piridina e $2 \theta=4,83^{\circ}$ para $n$ butilamina, calculou-se os valores das distâncias interlamelares, d, que foram de 11,04 e 18,27 $\AA$, respectivamente (Tabela 1). Os valores calculados conferem com os da literatura ${ }^{16,22}$. Observa-se que o aumento da distância interlamelar inicial do $\alpha-\mathrm{ZrP}$ de 7,53 para 11,04 e $18,27 \AA$ evidencia que houve intercalação da piridina. A Figura 3 dá uma idéia ilustrativa do possível arranjo da bicamada de n-butilamina entre as lamelas do fosfato de zircônio, como visto por Clearfield ${ }^{16}$.

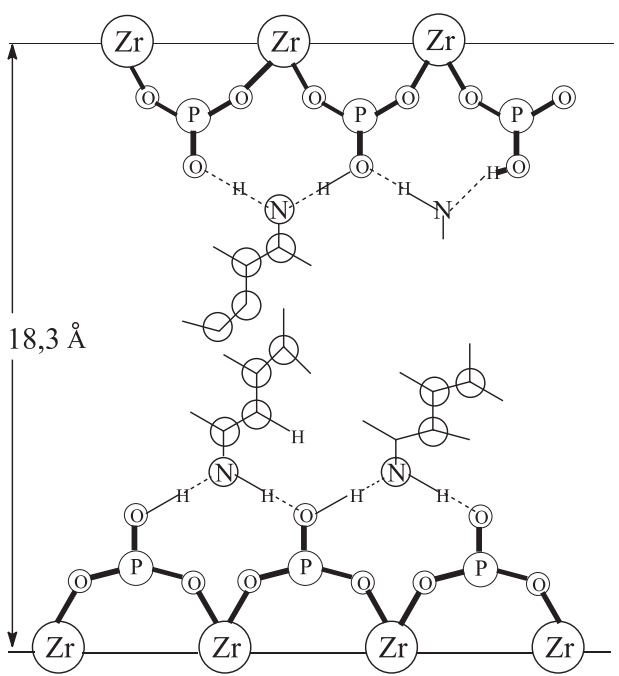

Figura 3. Esquema representativo da bicamada de n-butilamina em $\alpha-\mathrm{Zr}\left(\mathrm{HPO}_{4}\right)_{2}$ após intercalação

Segundo a literatura ${ }^{22,23}$, a intercalação do hidrogenofosfato de zircônio na forma alfa ocorre com a n-butilamina numa proporção maior que com a piridina. Enquanto $2 \mathrm{~mol}$ de n-butilamina são intercalados, somente $0,6 \mathrm{~mol}$ de piridina é intercalado. $\mathrm{O}$ comportamento de captação da piridina e n-butilamina pelo $\alpha-\mathrm{ZrP}$ pode ser interpretado em termos da presença de diferentes tipos de sítios ácidos no $\alpha-\mathrm{ZrP}$. A piridina é uma base fraca $(\mathrm{pKb}=8,8)^{24}$, para qual somente cerca de $25 \%$ dos sítios ácidos fortes do $\alpha$-ZrP estão disponíveis para ligação. A n-butilamina é uma base muito mais forte $(\mathrm{pKb}=3,4)^{25}$ que a piridina e, portanto, pode ocupar $67 \%$ dos sítios ácidos fortes do $\alpha-\mathrm{ZrP}^{22}$. A n-butilamina apesar de seu tamanho $(7,3 \AA)^{26}$ possui excelente mobilidade interlamelar, de modo que isso facilita o mecanismo de intercalação pela mudança de conformação das suas moléculas ${ }^{27}$. Por outro lado, para a piridina que embora seja menor $(3,5 \AA)^{26}$ comparada à n-butilamina, há a formação de apenas uma monocamada que é explicada em função da rigidez de sua estrutura.

A Figura 4 apresenta os espectros vibracionais na região do infravermelho para o $\alpha$-ZrP cristalino (Figura 4a), o $\alpha-\mathrm{ZrP}$ intercalado com piridina (Figura 4 b) e o $\alpha-\mathrm{ZrP}$ intercalado com $n-$ 
butilamina (Figura 4c). O espectro de infravermelho do $\alpha-\mathrm{ZrP}$ cristalino foi caracterizado pelas bandas em 3510 e $3593 \mathrm{~cm}^{-1}$, relacionadas aos modos vibracionais de estiramento do grupo $\mathrm{OH}$ livre ligado ao fósforo ${ }^{27,28}$.

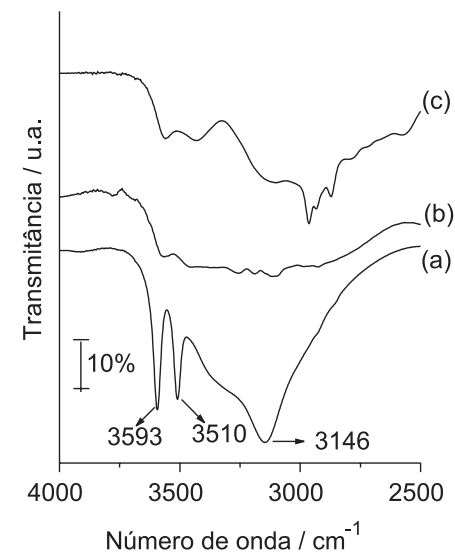

Figura 4. Espectros no infravermelho do hidrogenofosfato de zircônio (a) cristalino, (b) intercalado com piridina e (c) intercalado com n-butilamina

A constatação de que houve intercalação do $\alpha$-ZrP cristalino pela piridina e n-butilamina é feita ao se analisar e comparar entre si os espectros vibracionais na região do infravermelho da Figura 4. As bandas de estiramento em 3510 e $3593 \mathrm{~cm}^{-1}$, observadas no espectro do $\alpha-\mathrm{ZrP}$ cristalino (Figura $4 \mathrm{a}$ ), têem suas intensidades diminuídas após a intercalação com piridina e n-butilamina como pode-se observar nos espectros das Figuras $4 \mathrm{~b}$ e $4 \mathrm{c}$, respectivamente. A diminuição considerável na intensidade desses modos vibracionais, nessas regiões, deve-se à formação de ligação de hidrogênio, OH----N, entre os centros básicos da piridina e nbutilamina e $\mathrm{P}-\mathrm{OH}$ do grupo fosfato, comprovando-se assim o êxito das reações do $\alpha-Z r P$ cristalino com a piridina e com a nbutilamina, respectivamente.

\section{CONCLUSÃO}

Este experimento foi realizado por alunos do curso de química, na disciplina química inorgânica experimental, onde se procurou, com uso de uma linguagem simples, abordar temas relacionados com o estado sólido que, para a grande maioria dos químicos, se constitui numa área de conhecimento voltada quase que exclusivamente para a elucidação e compreensão de estrutura. Na química inorgânica estes aspectos de interesse são muito importantes, pela oportunidade de exploração de modelos idealizados e simples que explicam as ligações químicas e ficam muito mais interessantes quando se consegue sair do plano teórico, de sala de aula, para o universo grandioso de um laboratório de química.

Este trabalho teve o propósito de desenvolver a síntese do hidrogenofosfato de zircônio cristalino e alterar suas propriedades pela intercalação de aminas neutras.

A intercalação do hidrogenofosfato de zircônio cristalino com uma amina aromática, piridina, e outra alifática, n-butilamina, demonstrou que o comportamento de captação das mesmas, pelo $\alpha$ $\mathrm{ZrP}$, pode ser interpretado em termos da presença de diferentes tipos de sítios ácidos no $\alpha-\mathrm{ZrP}$ e da força básica das respectivas aminas. Desse modo, enquanto uma amina primária, n-butilamina, pode ocupar grande maioria dos sítios disponíveis, a piridina ocupou uma menor quantidade de sítios ativos do hidrogenofosfato de zircônio.

O uso da espectroscopia vibracional na região do infravermelho pode fornecer informações adicionais e comprobatórias àquelas obtidas por difração de raios-X, acrescentando conhecimentos adicionais pelo uso de técnicas distintas na caracterização de novos materiais.

\section{AGRADECIMENTOS}

W. S. Cardoso agradece à FUNCAMP pela concessão do estágio de capacitação docente junto ao IQ-UNICAMP e ao CNPq pela bolsa de doutoramento recebida.

\section{REFERÊNCIAS}

1. Sanches-Andujar, M.; Rivas-Murias, B; Rinaldi, D.;Caciuffo, R.; Mira, J.; Rivas, J.; Senaris-Rodriguez, M. A.; J. Magn. Magn. Mater. 2004, 855856, 272.

2. Jaiswal, A.; Collins, J.; Agricole, B.; Delhaes, P.; Ravaine, S.; J. Colloid Interface Sci. 2003, 261, 330.

3. Dresselhaus, M. S.; Dresselhaus, G.; Advances in Physics 2002, 51, 1.

4. Hiyoshi, N.; Yamamoto, N.; Kamiya, Y.; Nishikawa, E; Okuhara, T.; Catal. Today 2001, 71, 129.

5. Ana, M. A. S.; Benavente, E.; Gonzalez, G.; J. Coord. Chem. 2001, 54, 481.

6. Dines, M. B.; J. Chem. Educ. 1974, 51, 221

7. Schollhorn, R.; Physica B 1980, 98, 89.

8. Ubbelohde, A. R. Em Intercalated Layered Materials; Lévy, F. A.; ed.; D. Reidel Publishing Company: Dordrecht, 1979, cap. 1.

9. Bruce, D. W.; O'Hare, D.; Inorganic Materials, $2^{\text {nd }}$ ed., John Wiley \& Sons: Chichester, p. 171.

10. Schöllhorn, R. Em Inclusion Compounds - Structural Aspect of Inclusion Compounds Formed by Inorganic and Organometallic Host Lattices; Atwood, J. L.; Davies, J. E. D.; MacNicol, D. D., eds.; Academic Press Inc.: London, 1984, vol. 1.

11. Nunes, L. M.; Airoldi, C.; Quim. Nova 2001, 24, 799.

12. Zarbin, A. J. G.; Davanzo, C. U.; Quim. Nova 1995, 494.

13. Nakayama, H.; Hirami, S.; Tsuhako, M.; Chem. Lett. 2004, 33, 712.

14. Romano, R.; Ruiz. A. I.; Alves, O. L.; J. Solid State Chem. 2004, 177, 1520.

15. Li, W. Z.; Qin, C. G.; Lv, C. S.; Li, L. S.; Chen, J. S.; Catal. Lett. 2004, 94, 95.

16. Clearfield, A.; Inorganic Exchange Materials, CRC Press: Boca Raton, Florida, 1982, p. 112.

17. Clearfield, A. Em Comprehensive Supramolecular Chemistry; Alberti, G.; Bein, T., eds.; Pergamon: New York, 1996.

18. Behrendt, D.; Beneke, K.; Lagaly, G.; Angew. Chem., Int. Ed. 1976, 15, 544.

19. Alberti, G.; Torraca, E.; J. Inorg. Nucl. Chem. 1968, 30, 317.

20. Marttel, A. E.; Chemistry of the Metal Chelate Compounds, Prentice-Hall, Inc.: New York, 1952, p. 467.

21. Powder diffraction fill-search manual, Pensilvânia, USA, 1973.

22. Yamanaka, S.; Horibe, Y.; Tanaka, M.; J. Inorg. Nucl. Chem. 1976, 38, 323.

23. Clearfield, A.; Tindwa, R.M.; J. Inorg. Nucl. Chem. 1979, 41, 871.

24. Carey, F. A.; Organic Chemistry, $3^{\text {rd }}$ ed., McGraw-Hill: New York, 1996, p. 908.

25. Hart, H.; Schuetz, R. D.; Organic Chemistry: A short course, $4^{\text {th }}$ ed., Michigan State University: USA, 1972, p. 272.

26. Streitwieser, A.; Heathcock, C. H.; Kosower, E. M.; Introduction to Organic Chemistry, $4^{\text {th }}$ ed., MacMillan Publishing Co.: New York, 1992, p. 726.

27. MacLachlan, D. J.; Morgar, K. R.; J. Phys. Chem. 1992, 96, 3458.

28. Bellamy, L. J.; The Infra-Red Spectra of Complex Molecules, $2^{\text {nd }}$ ed., John Wiley and Sons, Inc.: New York, 1958. 